\title{
Effect of stimulus frequency and stimulation site on the N1m response of the human auditory cortex
}

\author{
D. Gabriel ${ }^{\mathrm{a}, *}$, E. Veuillet ${ }^{\mathrm{a}}$, R. Ragot ${ }^{\mathrm{b}}$, D. Schwartz ${ }^{\mathrm{b}}$, A. Ducorps ${ }^{\mathrm{b}}$, A. Norena ${ }^{\mathrm{a}}$, \\ J.D. Durrant ${ }^{\mathrm{a}}$, A. Bonmartin ${ }^{\mathrm{c}}$, F. Cotton ${ }^{\mathrm{c}}$, L. Collet ${ }^{\mathrm{a}}$ \\ a Unité CNRS UMR 5020, Laboratoire "Neurosciences et Systèmes Sensoriels", CNRS GDR 2213 "Prothèses Auditives", \\ Université Claude Bernard Lyon I, 50 av. Tony Garnier, 69366 Lyon, Cedex 07, France \\ ${ }^{\mathrm{b}}$ LENA CNRS UPR 640, Hôpital de la Salpêtrière, 47 Bd. de l'Hôpital, 75651 Paris, Cedex 13, France \\ ${ }^{c}$ Unité RMN, Centre hospitalier Lyon Sud, 165 Chemin du Grand Revoyet, 69495 Pierre-Benite, France \\ Received 16 March 2004; accepted 20 July 2004 \\ Available online 12 September 2004
}

\begin{abstract}
The aim of the present study was to investigate the functional organization of the auditory cortex for pure tones of $1,2,4,6,8$ and $12 \mathrm{kHz}$. Ten subjects were tested with a whole-head magnetometer ( 151 channels). The location, latency and amplitude of the generators of the $\mathrm{N} 1 \mathrm{~m}$ (the main component of the response, peaking approximately at $100 \mathrm{~ms}$ ) were explored simultaneously in the right and left hemispheres under monaural stimulation. Our results revealed that tonotopy is a rather complex functional organization of the auditory cortex. From 1 to $12 \mathrm{kHz}$, tonotopic maps were found for contralateral as well as for ipsilateral stimulation: $\mathrm{N} 1 \mathrm{~m}$ generators were found to be tonotopically organized mainly in an anterior-posterior direction in both hemispheres, whatever the stimulated ear, but also in an inferior-superior direction in the right hemisphere. Furthermore, latencies were longer in the left than in the right hemisphere. Two different representations of spectral distribution were found in the right auditory cortex: one for ipsilateral and one for contralateral stimulation.
\end{abstract}

(C) 2004 Elsevier B.V. All rights reserved.

Keywords: Tonotopy; Magnetoencephalography; Asymmetry; High frequencies

\section{Introduction}

Since the early 1960s (Bekesy, 1960), it has been known that sounds activate specific areas of the cochlea according to their frequency. The basal area of the coch-

Abbreviations: AEF, Auditory evoked fields; ECD, Equivalent current dipole; EEG, Electroencephalography; MRI, Functional magnetic resonance imaging; LHc, Contralateral stimulation in the left hemisphere; LHi, Ipsilateral stimulation in the left hemisphere; MEG, Magnetoencephalography; MRI, Magnetic resonance imaging; PET, Positron emission tomography; RHc, Contralateral stimulation in the right hemisphere; RHi, Ipsilateral stimulation in the right hemisphere

${ }^{*}$ Corresponding author. Tel.: +(33) 04372874 85; fax: +(33) 0437 287601 .

E-mail address: damiengabriel@yahoo.fr (D. Gabriel). lea codes high and the apical area low frequencies. This tonotopy also exists throughout the auditory pathway up to the primary auditory cortex. The first studies describing this cortical organization were made in monkeys (Merzenich and Brugge, 1973) and in cats (Merzenich et al., 1975).

In humans, the first investigations of the auditory cortex were hindered by the fact that the techniques used to record the cortical activity were invasive (e.g. Celesia, 1976).

A breakthrough in the study of cortical tonotopy came with the appearance and development of less or non-invasive measurement techniques: positron emission tomography (PET), functional magnetic resonance imaging (fMRI), electroencephalography (EEG), and 
magneto-encephalography (MEG). With stimulation frequencies between 250 and $6000 \mathrm{~Hz}$, most of the studies observed a tonotopic organization of the auditory cortex in an anterior-to-posterior direction, with high frequency tones represented more posterior and medial in the auditory cortex: e.g. Lauter et al. (1985) using PET; Wessinger et al. (1997) and Bilecen et al. (1998) using fMRI; Verkindt et al. (1995) using EEG; and Pantev et al. $(1988,1993)$ using MEG.

There are, however, very few studies describing the tonotopic organization of the auditory cortex for frequencies higher than $6000 \mathrm{~Hz}$, although these frequencies have been shown to convey important acoustic cues in speech recognition, especially for sibilant consonants (Behrens and Blumstein, 1988). Muhlnickel et al. (1998) demonstrated that the auditory evoked fields (AEF) for $8000 \mathrm{~Hz}$ tones were deeper in the auditory cortex than those for $6000 \mathrm{~Hz}$ tones. In a recent study, Fujioka et al. (2002) found a significant regression in the left hemisphere for tones of frequencies between 4000 and $15,000 \mathrm{~Hz}$ to be located more medially in proportion to the logarithm of stimulus frequency, but did not observe any significant difference in dipole locations for each tone frequency.

Another important feature in the tonotopy of A1 is the variability of the cortical maps according to the hemisphere studied and to the ear stimulated. It has been suggested, by intracerebral electrode studies (Liegeois-Chauvel et al., 2001), that the right hemisphere is more precisely organized than the left, but no neuromagnetic studies have yet clearly demonstrated this. A few MEG studies also noted discrepancies between right and left hemisphere. Indeed, Rosburg et al. (1998) found a tonotopic organization only in the left auditory cortex, and Fujioka et al. (2002) found a tendency, which did not, however, attain statistical significance, for the right hemisphere to be less highly organized than the left. The variation in cortical maps depending on the ear stimulated remains unknown, no studies, to our knowledge, having focused specifically on this topic yet.

Thus, the main goal of the present study was to describe accurately the tonotopic organization of the auditory cortex. First, we examined whether functional organization still exists for high frequency tones: i.e., higher than $6000 \mathrm{~Hz}$. Next, we examined the variability of the tonotopic maps, whether the hemispheres were differently organized, and whether the functional organization of the maps is affected by the ear stimulated.

\section{Materials and methods}

Ten healthy right-handed male subjects (aged between 20 and 25 years) participated in this study. All had normal hearing with no history of otological or neurological disorder. Informed consent was obtained from the partici- pants prior to the study and the subjects were paid for their participation. The experimental procedures were approved by an ethical committee in Lyon (France).

Auditory stimuli were tone bursts of $200 \mathrm{~ms}$ duration, with $3 \mathrm{~ms}$ rise and fall time. Stimuli were pure tones of 6 different frequencies: 1000, 2000, 4000, 6000, 8000 and $12,000 \mathrm{~Hz}$. For each frequency, intensity was set to 60 $\mathrm{dB}$ relative to the individual hearing threshold $(\mathrm{dB}$ $\mathrm{SL}$ ). The presentation of these six stimuli was varied pseudo-randomly (successive repetition of the same tone was avoided) with interstimulus interval varying randomly between 600 and $800 \mathrm{~ms}$. Seven hundred and fifty stimuli (125 of each frequency) constituted one train of stimuli. Four trains were recorded alternately in each ear, with 1-min rest pauses between trains.

Tones were generated by a piezo-electric transducer (with a linear bandwith from 2000 to $16,000 \mathrm{~Hz}$ ) located $1 \mathrm{~m}$ away from the subject's head, and were delivered through a plastic tube $(13 \mathrm{~mm}$ in inner diameter, $1 \mathrm{~m}$ long) into a specially designed polyester adapter with silicon ear tubing. Outside of the tube, the signal was analyzed for each frequency, and total harmonic distortions for each frequency were always lower than $0.01 \%$.

Magnetic fields were recorded in MEG Center of Paris with an Omega 2000 Whole Head System with 151 gradiometers (CTF, Vancouver). Sampling rate was $625 \mathrm{~Hz}$.

All neuromagnetic measurements were carried out within a magnetically shielded room, with the subject lying in a supine position. The ambient noise inside the room was always less than $60 \mathrm{~dB}$ SPL. In order to restrain head movement, the subject's head was held against the inner surface of the MEG recording system by means of a silicon helmet fitted by means of adjustable Velcro.

\subsection{Data analysis}

Analysis were first performed on the average of the two first trains of stimuli, i.e. the average of 250 recordings for each frequency. Epoch recordings of $890 \mathrm{~ms}$ length (with prestimulus time of $200 \mathrm{~ms}$ ) were filtered with a lowpass filter with a cutoff frequency of $0.1 \mathrm{~Hz}$. All recordings were visually inspected, and those contaminated by muscle or eye-blink artifacts were rejected before the averaging procedure. Next, the same analysis was performed on the average of the last two trains in order to assess the reproducibility of the experiment.

Source analysis was based on a single moving equivalent current dipole (ECD) model in a spherical volume conductor. Generators of the recorded response were modeled with a Single Moving Dipole Fit Algorithm. Multi-dipole models, taking into account the multiple sources of the generators in the auditory cortex, tended to be unstable and were consequently not used in our study.

The N1m wave was analyzed specifically in the channels corresponding to the hemisphere studied with 
respect to latency (time interval between the stimulus onset and the maximal Root Mean Square value of the N1m wave), amplitude (measured with Root Mean Square and with Dipole Moment), and the location of the ECD for ipsilateral and contralateral stimulation. All these parameters were studied at the peak of the $\mathrm{N} 1 \mathrm{~m}$ wave. The location of ECD was accepted if it was in agreement with the following two requirements:

1. The error function (calculated with a normalized least-square method and expressed as a percentage) in the fitting of the ECD in the recorded magnetic field had to be $<15 \%$.

The error equation was the following:

Error $=\frac{\sum_{j=1}^{M} \sum_{j=1}^{N}\left(B_{i j}-B_{i j}^{\prime}\right)^{2}}{\sum_{j=1}^{M} \sum_{j=1}^{N} B_{i j}^{2}}$,

with $N=$ number of sensors or electrodes; $M=$ number of time points in fit window; $B=$ observed signal at sensor $i ; B^{\prime}=$ calculated signal at sensor $i$.

2. In the $10 \mathrm{~ms}$ around the N1m peak, the dipole location must be stationary (variation less than $5 \mathrm{~mm}$ in each direction)

As MEG recordings are quite focal in each hemisphere, the two hemispheres were analyzed separately (in half of the channels), whichever the ear being stimulated: the moving dipole fit algorithm provides more reliable results when used in this way.

Consequently, the N1m wave was studied in four conditions:

- in the left hemisphere with contralateral (i.e., right ear) stimulation ( $\mathrm{LHc}$ ).

- in the left hemisphere with ipsilateral (i.e., left ear) stimulation (LHi).

- in the right hemisphere with contralateral stimulation (i.e., left ear) stimulation (RHc).

- in the right hemisphere with ipsilateral stimulation (i.e. right ear) stimulation (RHi).

During MEG recording, source location was estimated in a head-based coordinate system. In order to fix sensor positions relative to the head, three coils were positioned on the nasion and on the two pre-auricular points. The origin of this coordinate system was set at the midpoint of the medio-lateral axis ( $y$ axis) which connected the two pre-auricular points. The posterior-anterior axis $(x$ axis) was oriented from this origin to the nasion, and the inferior-superior axis ( $z$ axis) was perpendicular to the $x-y$ plane and pointing to the vertex.

\subsubsection{MRI recordings}

Magnetic resonance imaging (MRI) was performed in six subjects, using a 1.5-T scanner (Gyroscan Intera
Philips). Recordings were a 3D sagittal acquisition, with a repetition time of $24 \mathrm{~ms}$, an echo time of $8 \mathrm{~ms}$, and slice thickness of $0.98 \mathrm{~cm}$. During the MRI recording, three small capsules of vitamin E were fixed above the landmark sites on the head.

In order to match the MEG recordings with the MRI slices, three landmark sites were used during the MRI recording: one in the nasion (intersection between the nose and forehead), one in the left ear canal and one in the right ear canal. During the MEG experiment, coils were located on these landmarks, the location of these coils being recorded by the MEG system itself.

\subsubsection{Statistical analysis}

The posterior-anterior, medial-lateral and inferiorsuperior ECD locations, the RMS amplitude, the dipole moment and the latency of the N1m were analyzed separately in the above four N1m wave study conditions (LHc, LHi, RHc, RHi) with a one-way repeated measures ANOVA, the repeated measures factor being frequency $(1000,2000,4000,6000,8000$, and 12,000 Hz).

The comparisons between ipsi- and contra-lateral stimulation and between left and right hemisphere were made with a two-way repeated measures ANOVA, the repeated measures factor being frequency and either ear stimulated or hemisphere studied.

Post-hoc comparisons were made by Tukey test.

\section{Results}

- Functional organization in the left hemisphere (Table 1)

Two subjects did not provide reliable data in at least one of the six stimulation frequencies after contralateral stimulation, and were excluded from contralateral statistical analysis. For ipsilateral stimulation, the N1m wave was also difficult to map in at least one frequency in four subjects, who were excluded from the ipsilateral analysis.

Following contralateral stimulation ( $\mathrm{LHc}$ ), a tonotopic organization was observed in the anterior-posterior $(X)$ axis $[F(7,40)=3.978, p=0.006]$, the $1 \mathrm{kHz}$ generator of the $\mathrm{N} 1 \mathrm{~m}$ wave being located significantly more frontally in the primary cortex than the $12 \mathrm{kHz}$ generator $(p=0.006)$. This effect could be observed in all subjects individually, except one. A marginally significant organization was observed in the medial-lateral $(Y)$ axis $[F(7,40)=2.313, p=0.065]$. But no functional organization was observed between frequencies in the inferior-superior $(Z)$ plane.

$\mathrm{N} 1 \mathrm{~m}$ wave amplitude decreased gradually with increasing stimulus frequency $[F(7,47)=11.010$, $p<0.001$ for RMS; $F(7,40)=4.378, p=0.003$ for dipole moment]. 
Table 1

Latency, amplitude, dipole moment, and ECD location of N1m components and their corresponding standard deviation in the left hemisphere following right or left ear presentation of pure tones of six different frequencies $(1000,2000,4000,6000,8,000 \mathrm{and} 12,000 \mathrm{~Hz})$

\begin{tabular}{|c|c|c|c|c|c|c|c|}
\hline & \multirow[t]{2}{*}{$N$} & \multirow[t]{2}{*}{ Latency (ms) } & \multirow[t]{2}{*}{ Amplitude (fT) } & \multirow[t]{2}{*}{ Dip mom (nAm) } & \multicolumn{3}{|l|}{ ECD location } \\
\hline & & & & & $X(\mathrm{~cm})$ & $Y(\mathrm{~cm})$ & $Z(\mathrm{~cm})$ \\
\hline \multicolumn{8}{|c|}{ Left hemisphere } \\
\hline \multicolumn{8}{|c|}{ Contralateral stimulation } \\
\hline $1000 \mathrm{~Hz}$ & $8(10)$ & $105.25 \pm 5.47$ & $982.50 \pm 223.14$ & $46.10 \pm 16.97$ & $0.491 \pm 0.784$ & $5.271 \pm 0.191$ & $5.778 \pm 0.422$ \\
\hline $2000 \mathrm{~Hz}$ & $8(10)$ & $104.38 \pm 6.00$ & $795.63 \pm 150.01$ & $39.78 \pm 13.37$ & $0.308 \pm 0.789$ & $5.180 \pm 0.345$ & $5.545 \pm 0.640$ \\
\hline $4000 \mathrm{~Hz}$ & $8(10)$ & $100.75 \pm 5.85$ & $714.13 \pm 115.92$ & $35.18 \pm 9.47$ & $0.109 \pm 0.961$ & $5.213 \pm 0.256$ & $5.733 \pm 0.493$ \\
\hline $6000 \mathrm{~Hz}$ & $8(9)$ & $105.63 \pm 9.26$ & $631.38 \pm 117.50$ & $33.53 \pm 10.81$ & $-0.004 \pm 0.976$ & $5.065 \pm 0.434$ & $5.690 \pm 0.691$ \\
\hline $8000 \mathrm{~Hz}$ & $8(9)$ & $108.25 \pm 6.63$ & $600.25 \pm 131.38$ & $33.40 \pm 10.88$ & $0.203 \pm 0.859$ & $4.943 \pm 0.456$ & $5.893 \pm 0.676$ \\
\hline $12,000 \mathrm{~Hz}$ & $8(8)$ & $110.63 \pm 7.15$ & $573.88 \pm 109.32$ & $27.18 \pm 10.42$ & $-0.074 \pm 0.887$ & $5.270 \pm 0.460$ & $5.910 \pm 0.727$ \\
\hline \multicolumn{8}{|c|}{ Ipsilateral stimulation } \\
\hline $1000 \mathrm{~Hz}$ & $6(10)$ & $118.17 \pm 5.74$ & $806.67 \pm 211.13$ & $37.85 \pm 9.54$ & $0.80 \pm 0.71$ & $5.17 \pm 0.574$ & $5.582 \pm 0.517$ \\
\hline $2000 \mathrm{~Hz}$ & $6(10)$ & $117.83 \pm 4.62$ & $616.00 \pm 171.11$ & $28.85 \pm 14.35$ & $0.40 \pm 0.75$ & $5.41 \pm 0.710$ & $5.483 \pm 0.674$ \\
\hline $4000 \mathrm{~Hz}$ & $6(10)$ & $114.50 \pm 5.32$ & $534.83 \pm 151.53$ & $23.53 \pm 9.86$ & $0.52 \pm 0.65$ & $5.53 \pm 0.543$ & $5.710 \pm 0.814$ \\
\hline $6000 \mathrm{~Hz}$ & $6(9)$ & $117.83 \pm 11.77$ & $454.00 \pm 103.59$ & $20.68 \pm 6.88$ & $0.13 \pm 0.99$ & $5.42 \pm 0.404$ & $5.687 \pm 0.666$ \\
\hline $8000 \mathrm{~Hz}$ & $6(6)$ & $126.67 \pm 18.69$ & $419.67 \pm 78.23$ & $21.57 \pm 5.46$ & $0.36 \pm 0.84$ & $5.10 \pm 0.650$ & $5.720 \pm 0.764$ \\
\hline $12,000 \mathrm{~Hz}$ & $6(6)$ & $127.33 \pm 16.85$ & $377.00 \pm 94.06$ & $17.39 \pm 5.47$ & $0.42 \pm 0.85$ & $5.33 \pm 0.455$ & $5.787 \pm 0.803$ \\
\hline
\end{tabular}

$N$ is the number of subjects with an observable N1m in all six frequencies. In brackets are the number of subjects with an observable N1m for the corresponding stimulation frequency.

Following an ipsilateral stimulation (LHi), there was also a tonotopic organization in the anterior-posterior plane $[F(5,25)=2.668, p=0.047]$, with the $1 \mathrm{kHz}$ generator located more frontally than the $6 \mathrm{kHz}$ generator which was the most posterior generator.

The same amplitude effect was observed following contralateral stimulation; i.e., a decrease in amplitude with increasing stimulus frequency [RMS: $F(5,25)=18.155$, $p<0.001$; dipole moment : $F(5,25)=8.735 ; p<0.001]$.

Data of the seven subjects responding to ipsi- and contralateral stimulation from 1 to $6 \mathrm{kHz}$ tones were analyzed in order to demonstrate an effect of the side of stimulation (Table 3).

No differences in the location of the equivalent current dipoles (Fig. 1A) were observed in respect with the stimulated ear. However, latencies were significantly shorter $[F(1,35)=60.990 ; p<0.001]$ and amplitude significantly higher $[F(1,35)=11.287 ; p=0.015]$ after contralateral stimulation than after ipsilateral stimulation.

Furthermore, in the six subjects responding to ipsiand contralateral stimulation from 1 to $12 \mathrm{kHz}$, the same effect of amplitude could be observed for high frequencies, i.e. a significantly lower rms amplitude for ipsi than for contralateral stimulation [for rms amplitude: $F(1,5)=26.64 ; p=0.004$ ] (Fig. 4).

- Functional organization in the right hemisphere (Table 2)

With an ipsilateral stimulation, N1m ECD sources could not be modeled in at least one of the stimulation
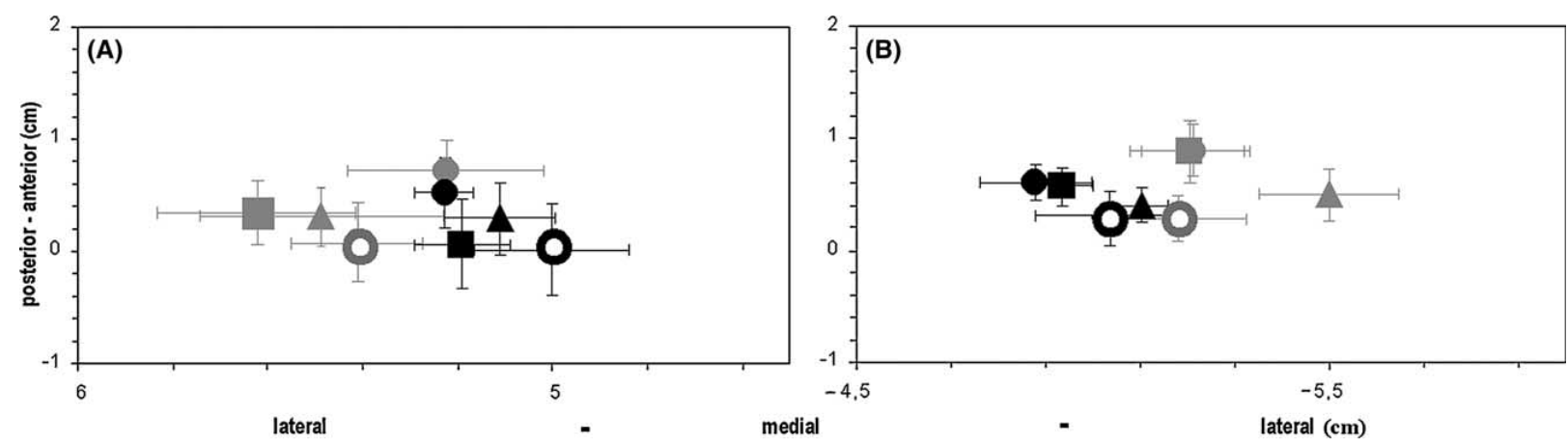

Fig. 1. Differences in the location of the N1m generators in the axial plane, according to stimulated ear. (A) Location of the generators in the left hemisphere, averaged from the seven subjects responding to both ipsilateral and contralateral stimulation for frequencies from 1 to $6 \mathrm{kHz}$. (B) Location of the generators in the right hemisphere, averaged from the nine subjects responding to both ipsilateral and contralateral stimulation for frequencies from 1 to $6 \mathrm{kHz}$. The N1m generators appearing with right ear stimulation are represented in black, and those appearing with left ear stimulation in grey. Error bars represent standard error. As there were no significant differences in the inferior-superior direction between LHc vs. $\mathrm{LHi}$ and between RHc vs. RHi, variation in this axis is not represented. 
Table 2

Latency, amplitude, dipole moment, ECD location of $\mathrm{N} 1 \mathrm{~m}$ components and their corresponding standard deviation in the right hemisphere following right or left ear presentation of pure tones of six different frequencies $(1000,2000,4000,6000,8000 \mathrm{and} 12,000 \mathrm{~Hz})$

\begin{tabular}{|c|c|c|c|c|c|c|c|}
\hline & \multirow[t]{2}{*}{$N$} & \multirow[t]{2}{*}{ Latency (ms) } & \multirow[t]{2}{*}{ Amplitude (fT) } & \multirow[t]{2}{*}{ Dip mom (nAm) } & \multicolumn{3}{|l|}{ ECD location } \\
\hline & & & & & $X(\mathrm{~cm})$ & $Y(\mathrm{~cm})$ & $Z(\mathrm{~cm})$ \\
\hline \multicolumn{8}{|c|}{ Right hemisphere } \\
\hline \multicolumn{8}{|c|}{ Contralateral stimulation } \\
\hline $1000 \mathrm{~Hz}$ & $10(10)$ & $102.90 \pm 7.96$ & $960.80 \pm 362.63$ & $48.54 \pm 21.65$ & $0.852 \pm 0.584$ & $-5.030 \pm 0.436$ & $5.426 \pm 0.827$ \\
\hline $2000 \mathrm{~Hz}$ & $10(10)$ & $101.80 \pm 9.26$ & $881.60 \pm 299.91$ & $44.48 \pm 18.28$ & $0.771 \pm 0.555$ & $-5.001 \pm 0.236$ & $5.450 \pm 0.741$ \\
\hline $4000 \mathrm{~Hz}$ & $10(10)$ & $101.00 \pm 11.06$ & $787.20 \pm 261.60$ & $38.58 \pm 17.23$ & $0.655 \pm 0.572$ & $-5.094 \pm 0.260$ & $5.641 \pm 0.643$ \\
\hline $6000 \mathrm{~Hz}$ & $10(10)$ & $97.20 \pm 7.70$ & $688.00 \pm 304.93$ & $35.51 \pm 20.02$ & $0.557 \pm 0.647$ & $-4.996 \pm 0.514$ & $5.781 \pm 0.680$ \\
\hline $8000 \mathrm{~Hz}$ & $10(10)$ & $102.20 \pm 7.60$ & $624.60 \pm 253.49$ & $29.65 \pm 15.31$ & $0.448 \pm 0.538$ & $-5.139 \pm 0.447$ & $5.995 \pm 0.651$ \\
\hline $12,000 \mathrm{~Hz}$ & $10(10)$ & $103.40 \pm 10.21$ & $624.50 \pm 229.87$ & $31.94 \pm 14.28$ & $0.225 \pm 0.995$ & $-4.831 \pm 0.442$ & $5.834 \pm 0.784$ \\
\hline \multicolumn{8}{|c|}{ Ipsilateral stimulation } \\
\hline $1000 \mathrm{~Hz}$ & $6(10)$ & $117.33 \pm 9.46$ & $738.17 \pm 207.00$ & $31.42 \pm 9.04$ & $1.443 \pm 0.599$ & $-5.258 \pm 0.237$ & $5.393 \pm 0.968$ \\
\hline $2000 \mathrm{~Hz}$ & $6(10)$ & $116.83 \pm 8.45$ & $624.33 \pm 227.01$ & $27.23 \pm 11.99$ & $1.332 \pm 0.419$ & $-5.310 \pm 0.581$ & $5.592 \pm 0.756$ \\
\hline $4000 \mathrm{~Hz}$ & $6(10)$ & $111.67 \pm 10.86$ & $617.50 \pm 119.12$ & $24.75 \pm 8.94$ & $1.103 \pm 0.473$ & $-5.502 \pm 0.446$ & $5.800 \pm 0.952$ \\
\hline $6000 \mathrm{~Hz}$ & $6(9)$ & $111.33 \pm 11.13$ & $572.33 \pm 130.88$ & $25.43 \pm 10.81$ & $0.765 \pm 0.387$ & $-5.313 \pm 0.570$ & $5.792 \pm 0.999$ \\
\hline $8000 \mathrm{~Hz}$ & $6(6)$ & $111.67 \pm 10.98$ & $486.17 \pm 125.34$ & $20.22 \pm 5.81$ & $0.724 \pm 0.238$ & $-5.528 \pm 0.530$ & $6.074 \pm 0.697$ \\
\hline $12,000 \mathrm{~Hz}$ & $6(6)$ & $107.17 \pm 11.81$ & $503.67 \pm 114.73$ & $20.95 \pm 6.81$ & $0.710 \pm 0.340$ & $-5.352 \pm 0.335$ & $6.180 \pm 0.604$ \\
\hline
\end{tabular}

$N$ is the number of subjects with an observable N1m in all six frequencies. In brackets is the number of subjects with an observable N1m for the corresponding stimulation frequency.

frequencies in four subjects. Therefore, these subjects were excluded from further RHi analysis.

With contralateral stimulation (RHc), neurons in the primary auditory cortex were organized tonotopically in the anterior-posterior $[F(9,45)=8.956, p<0.001]$ and in the inferior-superior $[F(9,45)=3.558, p=0.009]$ planes (Fig. 2C). In all our subjects but one, $12 \mathrm{kHz}$ generators were located more posteriorly than $1 \mathrm{kHz}$
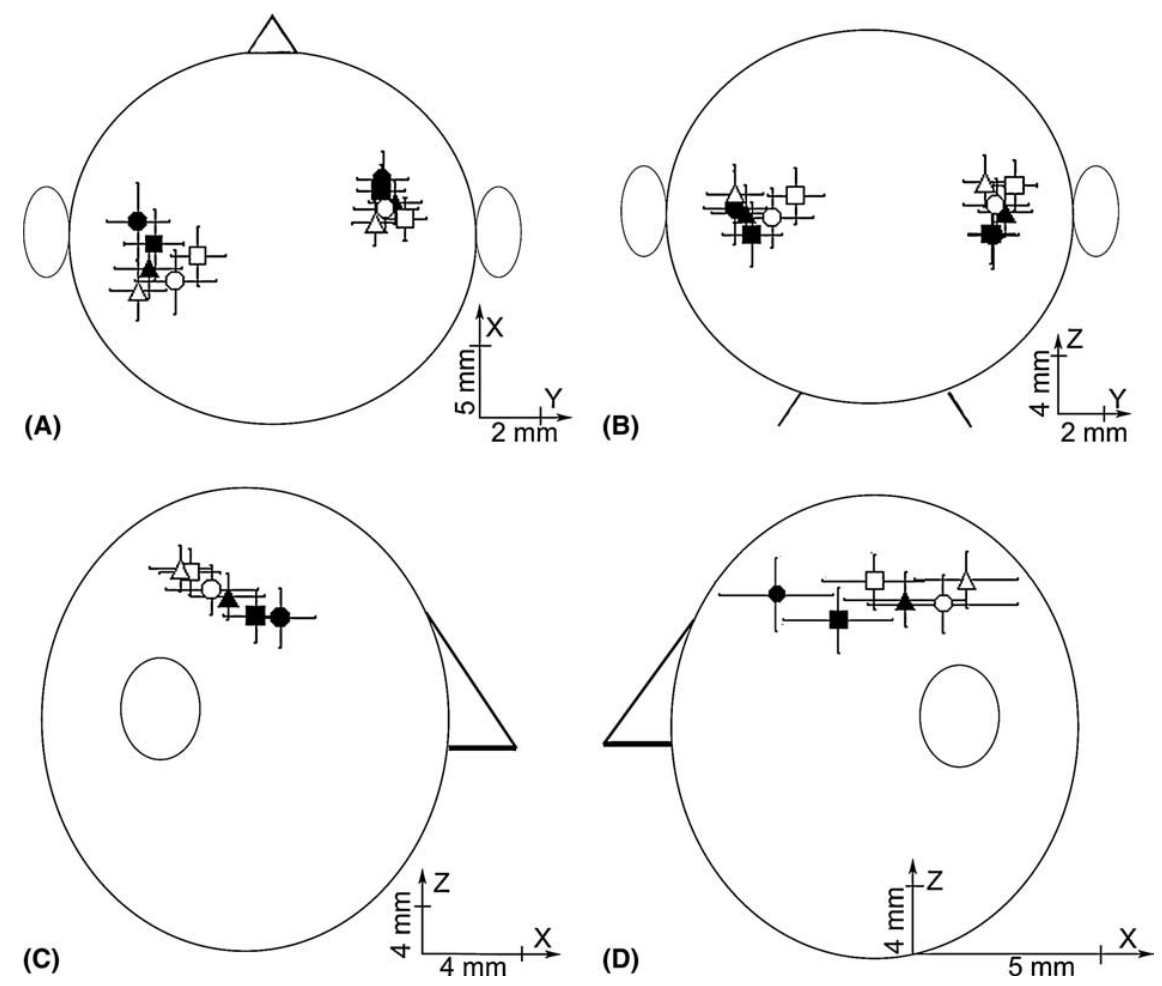

Fig. 2. Top (A), back (B), right (C) and left (D) views of the N1m wave generators following contralateral stimulation (LHc and RHc) in the eight subjects responding to every frequency. In the top view, the anterior-posterior variation of the dipoles is represented, as is the trend in the inferiorsuperior direction in the right side view. Error bars represent standard error. @: $1000 \mathrm{~Hz}$ generator; $\mathbf{\square}: 2000 \mathrm{~Hz} ; \mathbf{\Lambda}: 4000 \mathrm{~Hz} ; \bigcirc: 6000 \mathrm{~Hz}$; $\square: 8000$ $\mathrm{Hz} ; \triangle: 12,000 \mathrm{~Hz}$ 
Table 3

Equivalent current dipole location in the auditory cortex of subjects with an observable N1m from 1000 to $6000 \mathrm{~Hz}$ whatever the stimulated ear

\begin{tabular}{|c|c|c|c|c|c|c|c|c|}
\hline & \multicolumn{8}{|c|}{ ECD location } \\
\hline & \multicolumn{4}{|c|}{ Contralateral stimulation } & \multicolumn{4}{|c|}{ Ipsilateral stimulation } \\
\hline & $N$ & $\begin{array}{l}X(\mathrm{~cm}) \\
(\text { mean } \pm \mathrm{SD})\end{array}$ & $\begin{array}{l}Y(\mathrm{~cm}) \\
(\text { mean } \pm \mathrm{SD})\end{array}$ & $\begin{array}{l}Z(\mathrm{~cm}) \\
(\text { mean } \pm \mathrm{SD})\end{array}$ & $N$ & $\begin{array}{l}X(\mathrm{~cm}) \\
(\text { mean } \pm \mathrm{SD})\end{array}$ & $\begin{array}{l}Y(\mathrm{~cm}) \\
(\text { mean } \pm \mathrm{SD})\end{array}$ & $\begin{array}{l}Z(\mathrm{~cm}) \\
(\text { mean } \pm \mathrm{SD})\end{array}$ \\
\hline \multicolumn{9}{|c|}{ Right hemisphere } \\
\hline $1000 \mathrm{~Hz}$ & $9(10)$ & $0.600 \pm 0.496$ & $-4.880 \pm 0.351$ & $5.436 \pm 0.997$ & $9(10)$ & $0.893 \pm 0.684$ & $-5.211 \pm 0.323$ & $5.320 \pm 0.955$ \\
\hline $2000 \mathrm{~Hz}$ & $9(10)$ & $0.569 \pm 0.515$ & $-4.934 \pm 0.205$ & $5.599 \pm 0.800$ & $9(10)$ & $0.880 \pm 0.826$ & $-5.206 \pm 0.378$ & $5.333 \pm 0.710$ \\
\hline $4000 \mathrm{~Hz}$ & $9(10)$ & $0.400 \pm 0.457$ & $-5.104 \pm 0.165$ & $5.681 \pm 0.753$ & $9(10)$ & $0.493 \pm 0.709$ & $-5.499 \pm 0.439$ & $5.516 \pm 0.924$ \\
\hline $6000 \mathrm{~Hz}$ & $9(10)$ & $0.309 \pm 0.615$ & $-5.026 \pm 0.445$ & $5.829 \pm 0.666$ & $9(9)$ & $0.284 \pm 0.603$ & $-5.180 \pm 0.433$ & $5.611 \pm 0.870$ \\
\hline \multicolumn{9}{|c|}{ Left hemisphere } \\
\hline $1000 \mathrm{~Hz}$ & $7(10)$ & $0.521 \pm 0.841$ & $5.229 \pm 0.160$ & $5.823 \pm 0.435$ & $7(10)$ & $0.723 \pm 0.685$ & $5.224 \pm 0.546$ & $5.791 \pm 0.728$ \\
\hline $2000 \mathrm{~Hz}$ & $7(10)$ & $0.297 \pm 0.852$ & $5.110 \pm 0.305$ & $5.570 \pm 0.687$ & $7(10)$ & $0.319 \pm 0.712$ & $5.487 \pm 0.677$ & $5.736 \pm 0.908$ \\
\hline $4000 \mathrm{~Hz}$ & $7(10)$ & $0.067 \pm 1.031$ & $5.190 \pm 0.267$ & $5.803 \pm 0.488$ & $7(10)$ & $0.346 \pm 0.758$ & $5.623 \pm 0.555$ & $5.907 \pm 0.908$ \\
\hline $6000 \mathrm{~Hz}$ & $7(9)$ & $0.013 \pm 1.054$ & $5.001 \pm 0.427$ & $5.807 \pm 0.655$ & $7(9)$ & $0.080 \pm 0.917$ & $5.411 \pm 0.370$ & $6.016 \pm 1.062$ \\
\hline
\end{tabular}

$N$ is the number of subjects with an observable N1m. In brackets is the number of subjects with an observable N1m for the corresponding stimulation frequency.

generators, and two subjects only did not have $12 \mathrm{kHz}$ generators located more superiorly than $1 \mathrm{kHz}$ generators. N1m amplitude was significantly lower with high frequency tones than with low frequencies $[F(9,45)=7.885$, $p<0.001]$, especially 1 and $2 \mathrm{kHz}$ vs. 8 and $12 \mathrm{kHz}$ tones.

Ipsilateral stimulation also generates a tonotopic organization in the anterior-posterior $[F(5,25)=4.824$, $p=0.003]$ and in the inferior-superior $[F(5,25)=5.838$, $p=0.001)$ ] directions. The $\mathrm{N} 1 \mathrm{~m}$ amplitude was gradually decreasing with increasing stimulus frequency $[F(5,25)=9.958, p<0.001]$ and the amplitude of 1 $\mathrm{kHz}$ tones was significantly higher than all others stimulus frequencies amplitudes.

A comparison of differences in location, latency and amplitude between an ipsi- and contralateral stimulation was made on the nine subjects who had observable N1m from 1 to $6 \mathrm{kHz}$ (Table 3). N1m generators were not located at the same place with respect to the stimulation: there was a tendency for contralateral generators to be located more frontally than ipsilateral generators $[F(1,45)=5.046 ; p=0.055]$, post-hoc Tukey test revealing the difference to be significant for $1 \mathrm{kHz}$ $(p=0.045)$ and $2 \mathrm{kHz}$ tones $(p=0.043)$. But the generators were also significantly located more towards the right $[F(1,45)=14.871 ; p=0.005]$ for $2,4 \mathrm{kHz}$ and $6000 \mathrm{~Hz}$ tones, with a trend for the $1 \mathrm{kHz}$ tone (Fig. 1B).

In the six subjects responding to ipsi- and contralateral stimulation from 1 to $12 \mathrm{kHz}$, a clear effect of amplitude could be observed for high frequency tones, with an higher rms amplitude $[F(1,5)=9.019$; $p=0.03]$ for contralateral than for ipsilateral stimulation (Fig. 4).

- Differences between hemispheres

We compared left and right hemispheres in the eight subjects who had an observable N1m in every frequency after contralateral stimulation (Fig. 2). There was a marginally significant difference in the location of generators in the anterior-posterior direction between the right and the left hemisphere $[F(1,7)=4.348 ; p=0.076]$. Planned comparisons revealed this to be especially true for 1 $\mathrm{kHz}(p=0.08), 2 \mathrm{kHz}(p=0.032), 6 \mathrm{kHz}(p=0.091)$ and $12 \mathrm{kHz}(p=0.057)$ tones.

An example of the location of the ECD in one subject is given in Fig. 3.

A trend for latencies to be shorter in the right than in the left hemisphere was found $[F(1,35)=4.853$; $p=0.063]$. In order to study the impact of high frequency tones on these latency differences, planned comparisons were performed. Results indicated that latencies were significantly longer in the left hemisphere for tones of $6 \mathrm{kHz}[F(1,7)=12.790 ; p=0.009]$ and 12

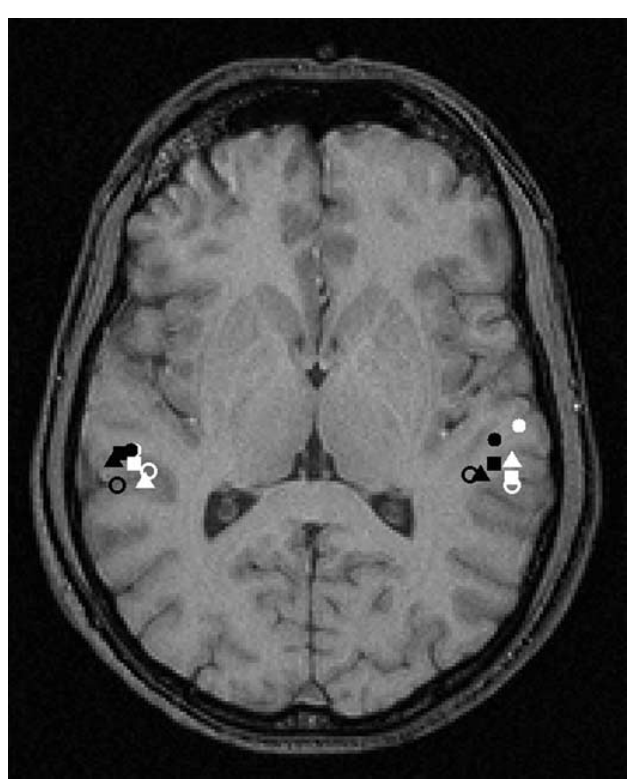

Fig. 3. Axial view of the location of the ECD from 1000 to $6000 \mathrm{kHz}$ in one subject. Filled circle: $1000 \mathrm{~Hz}$ generator. $\mathbf{\square}: 2000 \mathrm{~Hz} ; \mathbf{\Lambda}: 4000 \mathrm{~Hz}$; ○: $6000 \mathrm{~Hz}$. Right ear stimulation: light. Left ear stimulation: dark. 
$\mathrm{kHz}[F(1,7)=5.727 ; p=0.047]$, with a tendency for 8 $\mathrm{kHz}$ tones $[F(1.7)=4.225 ; p=0.079]$.

\section{Discussion}

\subsection{Latency and amplitude}

Roberts and Poeppel (1996) found that N1m wave latency varied with respect to stimulus frequency. Consequently, in the present study one would expect increased $\mathrm{N} 1 \mathrm{~m}$ latency with increasing stimulus frequency for tones higher than $1 \mathrm{kHz}$. However, as in the studies by Naka et al. (1999) and Fujioka et al. (2002), no such correlation was observed, and at all the frequencies tested in our study, no significant relation between N1m latency and stimulus frequency was found, whichever the ear stimulated. Thus, Roberts and Poeppel's (1996) finding of increased N1m latency with increasing stimulus frequency for tones higher than $1 \mathrm{kHz}$ was not confirmed. It is hard to explain these differences between Roberts and Poeppel's results and those of other studies including ours. The hypothesis suggested by Fujioka et al., who attributed the observed differences to differences in type of stimulation (binaural vs. monaural) or in stimulus frequency (low vs. high), cannot explain this, as we used monaural stimulation and the frequencies tested began at $1 \mathrm{kHz}$. An alternative hypothesis might be in terms of stimulus intensity. Roberts and Poeppel's intensity was set to the same sound pressure level whatever the frequency, which did not take account of the
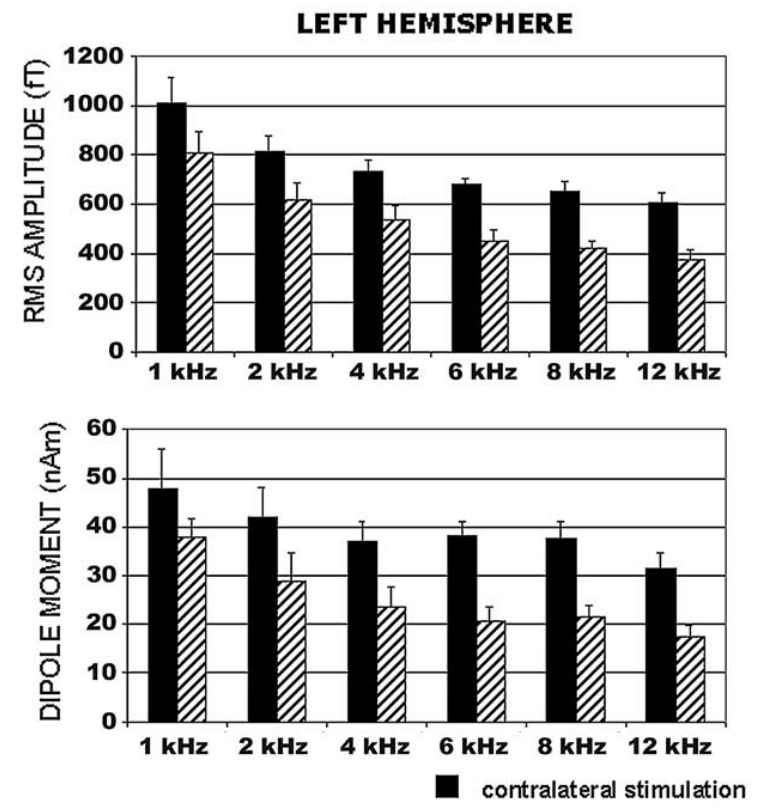

hearing threshold of the subjects and may consequently have been biased by possible cases of hearing loss. In our study, stimulation was at $60 \mathrm{~dB}$ above the individual hearing threshold, so that every frequency level used was somewhat normalized.

N1m amplitude and dipole moment gradually decreased as stimulus frequency increased. This decrease was especially observable between 1 and $6 \mathrm{kHz}$; for higher frequencies, amplitude remained low. A first hypothesis to explain these results could be that, as frequency increases between 1 and $6 \mathrm{kHz}$, less and less impulses from cochlear neurons succeed in remaining phase-locked with the incoming stimulus, and only the place coding remains operative. Another possible explanation of this decrease is that the ECD may be located more and more deeply in the auditory cortex as stimulus frequency increases; however, this would not agree with our results on tonotopic organization, since the dipole only shifted more posteriorly, and not more deeply, with increasing stimulus frequency (see below). Therefore, unlike Fujioka et al.'s (2002) results, ours do not suggest the existence of a barrier between tones lower and tones higher than $6 \mathrm{kHz}$. We may rather hypothesize that tones above $6 \mathrm{kHz}$ only activate a supraliminary $\mathrm{N} 1 \mathrm{~m}$, so that, if the tones had generated an AEF of lower amplitude, no N1m might have been recorded.

\subsection{Tonotopic organization of the auditory cortex}

Several studies have already focused on the tonotopic organization of the auditory cortex for middle or low
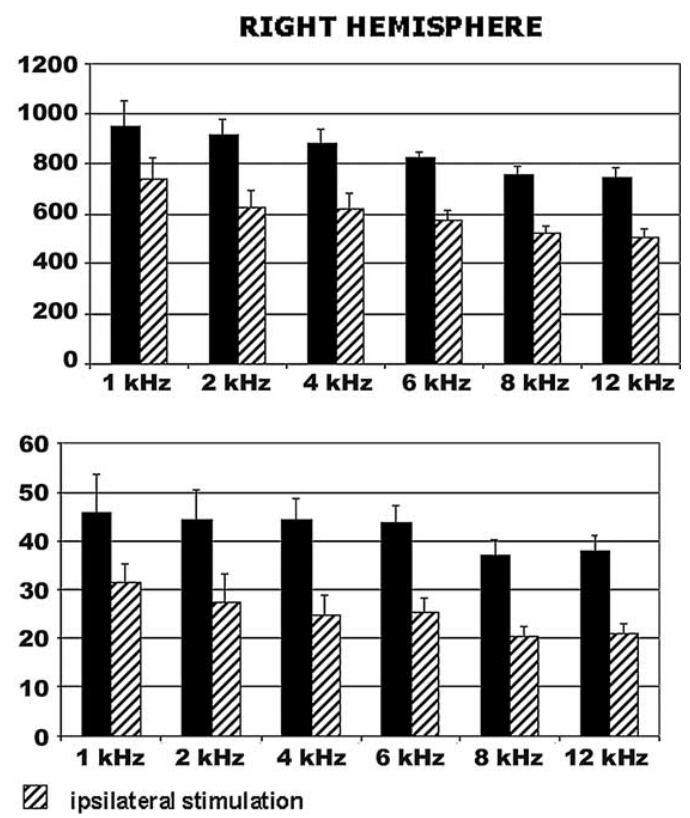

Fig. 4. Ipsilateral-contralateral comparisons for rms amplitude and dipole moment of the N1m wave in the six subjects responding to both ipsilateral and contralateral stimulation from 1 to $12 \mathrm{kHz}$ in the left hemisphere, and in the six subjects responding to both ipsilateral and contralateral stimulation in the right hemisphere. 
frequency waves up to $5 \mathrm{kHz}$. Concerning the N1m generators, most results have indicated a clear medio-lateral tendency, with activation of structures deeper in the auditory cortex with increasing stimulus frequency. However, such an organization remains controversial, as it seems not to be observed consistently in all subjects (Lütkenhöner et al., 2003).

The four conditions of our study ( $\mathrm{LHc}$, LHi, RHc, and RHi) revealed a tonotopic auditory cortex organization for high frequency tones, although not in the medio-lateral direction. In the left hemisphere, this organization was only significant in the anterior-posterior direction, with high frequency tones being located more posteriorly than low frequency tones. In the right hemisphere, high frequency tones were more posterior but also more superior than low frequencies. For contralateral stimulation, our results did not necessarily contradict those of previous studies. In the anteriorposterior direction, we found differences in N1m generator location between low (up to $4 \mathrm{kHz}$ ) and high frequency tones (more than $6 \mathrm{kHz}$ ), whereas the majority of previous studies did not describe any such difference, as they explored only low and medium frequencies. Furthermore, in the left hemisphere, a trend to lateral-to-medial functional organization after right ear stimulation was also observed. In case of ipsilateral stimulation, there are a very small number of studies which have as yet focused on tonotopy. Whereas PET techniques have proved unable to find any tonotopic organization in the ipsilateral hemisphere (Lauter et al., 1985; Lockwood et al., 1999), EEG and fMRI measurements revealed similar organization in right and left hemispheres after right ear stimulation (Verkindt et al., 1995; Strainer et al., 1997). Our results are not totally in agreement here, suggesting rather that ipsilateral maps are closer to contralateral maps in the same hemisphere, since ipsi- and contra-lateral righthemisphere maps showed the same inferior-superior tonotopic organization.

The anatomic location of the N1m generators is still a matter of debate in spite of all the MEG studies on the topic. Several studies have suggested that N1m originates from Heschl's Gyrus (Elberling et al., 1982; Reite et al., 1994); but the secondary auditory areas in the supratemporal plane are another possibility (Pantev et al., 1995; Lütkenhöner and Steinsträter, 1998). It would be useful to compare the location of these activation sites with cyto-architectonic data in order to assess whether frequency-dependent activated areas are located in the same physiologically defined field, which would reflect tonotopy, or in separate fields, which would not point to any tonotopic organization (Schönwiesner et al., 2002). However, a single tonotopically organized generator in the auditory cortex should not be too readily assumed, as there is growing evidence for several sources within the auditory cortex generating the N1m (Williamson et al., 1991; Lütkenhöner and Steinsträter, 1998). Moreover, Cansino et al. (1994) found another N1m tonotopic organization in the association cortex, although N1m might also originate from parietal (Lavikaïnen et al., 1994) or frontal (Giard et al., 1994) areas.

The major problem arising in all these studies, including our own, concerns the interpretation of this dipole organization. As the N1m wave clearly emerges from multiple sources in the auditory cortex (Naatanen and Picton, 1987), what help is a single moving dipole model regarding tonotopy? This question is all the more important in that multiple dipole models tend to be unstable and consequently inadequate. This problem is not a new one, and several studies have already noted that this single dipole approximation is a major limitation (Hari, 1990; Schreiner, 1998), and thus questioned on the use of magnetoencephalography in tonotopy (Lütkenhöner et al., 2003).

In order to propose an answer to this question, it may be useful to focus briefly on studies in animals. Microelectrode studies of non-human primates have provided many clues regarding the tonotopy of the auditory cortex and certainly give a good idea of how the human auditory cortex may be functionally organized. These studies tend to demonstrate the existence of at least seven tonotopic areas in the auditory cortex (Kosaki et al., 1997), with two or three being located in the primary auditory cortex (Kaas and Hackett, 2000). All these observations are made with numerous (up to 100) microelectrodes implanted in the auditory cortex. These results are clearly not definitive, and new frequencyspecific regions may be discovered soon with improvements the accuracy of recording techniques. In human studies, the spatial resolution of non-invasive recording techniques is insufficient for clear comparison, and intracerebral recording are limited in the number of microelectrodes that can be implanted. Consequently, one may assume that, whatever the recording technique used, tonotopy studies in human subjects certainly lack accuracy and may fail to observe some frequencydependent areas (for example, in MEG: Lütkenhöner et al., 2003; in fMRI: Schönwiesner et al., 2002).

In the present study, and also in all MEG studies with single dipole model analysis, two hypotheses can be made about dipole location and variation in the auditory cortex:

(1) The recorded N1m is generated by one predominant generator (i.e. an area with more activated neurons in it or nearer the scalp surface) and a few activation areas of less importance. In that ideal case, the dipole location and variation would be mainly based on the predominant generator location and thus the single dipole model would reflect one major tonotopic map. 
(2) The N1m wave is the result of the activation of several frequency-dependent areas of more or less equal importance. The location of the equivalent current dipole would thus be simply the center of mass of all these areas, and would have no physiological reality. The crucial point, however, concerns the variation in dipole location. Indeed, even if all of these generators exhibit their frequency-dependence in the generation of the N1m wave, it may be assumed that the variation in dipole location reflects an overall tonotopy for the N1m.

Consequently, whichever of these hypotheses is the right one, the observations made in our study can be explained by the fact that, for low frequency tones, the frequency-dependent areas of the generators coding the $\mathrm{N} 100 \mathrm{~m}$ are mainly located anteriorly, whereas with increasing stimulus frequency these areas as a whole shift in a more posterior direction.

\subsection{Several tonotopic maps?}

Pantev et al. (1998) observed a small difference in the location of $\mathrm{N} 1 \mathrm{~m}$ sources between ipsilateral and contralateral stimulation. Our study confirms these results and shows that, in the right hemisphere of the auditory cortex, two different tonotopic maps of the N1m could indeed coexist. Two hypotheses can be put forward to explain these results. First of all, just as the fiber trajectory is not the same for ipsilateral and for contralateral stimulation, as seen in the differences in latency, it may also be that the neurons activated by these two kinds of stimulation are different. Another hypothesis could be that, as there are multiple sources generating the $\mathrm{N} 1 \mathrm{~m}$, more generators could be activated with contralateral than with ipsilateral stimulation or only the relative amplitude of the underlying sources. With the approximation of one single current equivalent dipole, a shift in the location of the dipole may be observed. In addition to demonstrating that tonotopic mapping of pure tones extends to high frequencies, the present results thus suggest that the auditory cortex has two representations of the cochlea, at least in the right cortex: one for ipsilateral and one for contralateral stimulation.

\subsection{Differences between hemispheres}

One of the major drawbacks in MEG studies of neural asymmetry was that the first neuromagnetometers could not record brain activity in both hemispheres simultaneously. Recording one hemisphere at a time increases such disruptive effects as fatigue or altered vigilance. Nowadays, with whole-head neuromagnetometers, this no longer applies, and tonotopic functional organization laterality can be assessed with precision. Several studies have found functional asymmetry in auditory processing. For example, right hemisphere N1m generators are of greater amplitude (Kanno et al., 1996) and cover a larger area (Zouridakis et al., 1998) in the auditory cortex than do left hemisphere N1m generators. In the present study, N1m source location was more anterior in the right than in the left hemisphere, which is in agreement with previous whole-head MEG studies (Mäkelä et al., 1993; Nakasato et al., 1995; Pantev et al., 1998). This observation can be explained by the fact that the left temporal plane of right-handed subjects is significantly more posterior than the right (Geschwind and Levitsky, 1968; Szikla et al., 1977). Furthermore, as explained above, tonotopic organization differs between hemispheres, with only an anterior-to-posterior tonotopic map in the left hemisphere, whereas there is both an anterior-to-posterior and an inferior-to-superior map in the right. Moreover, we observed that latencies were longer in the left than in the right hemisphere, although this was just a trend. One possible explanation could be that the above-mentioned anatomical asymmetry of the temporal lobes is correlated with longer ascending pathways. Anatomic studies, however, are needed to investigate this hypothesis. However, it should be noted that our study was only performed in right-handed male subjects, and that hemispheric differences may not be the same in female or in left-handed subjects.

In conclusion, new clues are provided as to the tonotopic organization of the auditory cortex in humans. Firstly, high frequency tones are encoded more posteriorly than low frequency tones. Secondly, functional differences are found between A1 hemispheres, with generator location varying in antero-posterior and infero-superior directions in the right hemisphere, whereas in the left hemisphere such a tonotopic gradient was observed only in the antero-posterior direction. Thirdly, the right auditory cortex would seem to encode several tonotopic maps, depending on which ear is stimulated.

\section{Acknowledgement}

The study was supported by grants from CCA Groupe-Entendre-Oticon-Phonak-Siemens Audiology.

\section{References}

Behrens, S., Blumstein, S.E., 1988. Acoustic characteristics of English voiceless fricatives: A description analysis. J. Phonetics 16, 295-298. Bekesy, G. von, 1960. Experiments in Hearing. McGraw Hill Book Co., New York.

Bilecen, D., Scheffler, K., Schmid, N., Tschopp, K., Seelig, J., 1998. Tonotopic organization of the human auditory cortex as detected by BOLD- FMRI. Hear. Res. 126, 19-27.

Cansino, S., Williamson, S.J., Karron, D., 1994. Tonotopic organization of human auditory association cortex. Brain Res. 663, 38-50. Celesia, G.G., 1976. Organization of auditory cortical areas in man. Brain 99, 403-414. 
Elberling, C., Bak, C., Kofoed, B., Lebech, J., Saermark, K., 1982. Auditory magnetic fields: source location and 'tonotopical organization' in the right hemisphere of the human brain. Scand. Audiol. $11,61-65$.

Fujioka, T., Kakigi, R., Gunji, A., Takeshima, Y., 2002. The auditory evoked magnetic fields to very high frequency tones. Neuroscience 112, 367-381.

Geschwind, N., Levitsky, W., 1968. Human brain: left-right asymmetries in temporal speech region. Science 161, 186-187.

Giard, M.H., Perrin, F., Echallier, J.F., Thevenet, M., Froment, J.C., Pernier, J., 1994. Dissociation of temporal and frontal components in the human auditory N1 wave: a scalp current density and dipole model analysis. Electroencephalogr. Clin. Neurophysiol. 92, 238-252.

Hari, R., 1990. Magnetic evoked fields of the human brain: basic principles and applications. Electroencephalogr. Clin. Neurophysiol. Suppl. 41, 3-12.

Kaas, J.H., Hackett, T.A., 2000. Subdivisions of auditory cortex and processing streams in primates. Proc. Natl. Acad. Sci. USA. 97, 11793-11799.

Kanno, A., Nakasato, N., Fujita, S., Seki, K., Kawamura, T., Ohtomo, S., Fujiwara, S., Yoshimoto, T., 1996. Right hemispheric dominance in the auditory evoked magnetic fields for pure-tone stimuli. Electroencephalogr. Clin. Neurophysiol. Suppl. 47, 129-132.

Kosaki, H., Hashikawa, T., He, J., Jones, E.G., 1997. Tonotopic organization of auditory cortical fields delineated by parvalbumin immunoreactivity in macaque monkeys. J. Comp. Neurol. 386, 304-316.

Lauter, J.L., Herscovitch, P., Formby, C., Raichle, M.E., 1985. Tonotopic organization in human auditory cortex revealed by positron emission tomography. Hear. Res. 20, 199-205.

Lavikainen, J., Huotilainen, M., Pekkonen, E., Ilmoniemi, R.J., Naatanen, R., 1994. Auditory stimuli activate parietal brain regions: a whole-head MEG study. Neuroreport 6, 182-184.

Liegeois-Chauvel, C., Giraud, K., Badier, J.M., Marquis, P., Chauvel, P., 2001. Intracerebral evoked potentials in pitch perception reveal a functional asymmetry of the human auditory cortex. Ann. NY Acad. Sci. 930, 117-132.

Lockwood, A.H., Salvi, R.J., Coad, M.L., Arnold, S.A., Wack, D.S., Murphy, B.W., Burkard, R.F., 1999. The functional anatomy of the normal human auditory system: responses to 0.5 and $4.0 \mathrm{kHz}$ tones at varied intensities. Cereb. Cortex. 9, 65-76.

Lütkenhöner, B., Steinstrater, O., 1998. High-precision neuromagnetic study of the functional organization of the human auditory cortex. Audiol. Neurootol. 3, 191-213.

Lütkenhöner, B., Krumbholz, A., Seither-Preisler, A., 2003. Studies of tonotopy based on wave N100 of the auditory evoked field are problematic. Neuroimage 19, 935-949.

Mäkelä, J.P., Ahonen, A., Hämäläinen, M., Hari, R., Ilmoniemi, R., Kajola, M., Knuutila, J., Lounasmaa, O.V., McEvoy, L., Salmelin, R., et al., 1993. Functionnal differences between auditory cortices of the two hemispheres revealed by whole-head neuromagnetometic recordings. Hum. Brain Mapp. 1, 48-56.

Merzenich, M.M., Brugge, J.F., 1973. Representation of the cochlear partition of the superior temporal plane of the macaque monkey. Brain Res. 50, 275-296.

Merzenich, M.M., Knight, P.L., Roth, G.L., 1975. Representation of cochlea within primary auditory cortex in the cat. J. Neurophysiol. 38, 231-249.

Muhlnickel, W., Elbert, T., Taub, E., Flor, H., 1998. Reorganization of auditory cortex in tinnitus. Proc. Natl. Acad. Sci. USA 95, $10340-10343$.
Naatanen, R., Picton, T., 1987. The N1 wave of the human electric and magnetic response to sound: a review and an analysis of the component structure. Psychophysiology 24, 375-425.

Naka, D., Kakigi, R., Hoshiyama, M., Yamasaki, H., Okusa, T., Koyama, S., 1999. Structure of the auditory evoked magnetic fields during sleep. Neuroscience 93, 573-583.

Nakasato, N., Fujita, S., Seki, K., Kawamura, T., Matani, A., Tamura, I., Fujiwara, S., Yoshimoto, T., 1995. Functional localization of bilateral auditory cortices using an MRI- linked whole head magnetoencephalography (MEG) system. Electroencephalogr. Clin. Neurophysiol. 94, 183-190.

Pantev, C., Hoke, M., Lehnertz, K., Lutkenhoner, B., Anogianakis, G., Wittkowski, W., 1988. Tonotopic organization of the human auditory cortex revealed by transient auditory evoked magnetic fields. Electroencephalogr. Clin. Neurophysiol. 69, 160-170.

Pantev, C., Elbert, T., Makeig, S., Hampson, S., Eulitz, C., Hoke, M., 1993. Relationship of transient and steady-state auditory evoked fields. Electroencephalogr. Clin. Neurophysiol. 88, 389-396.

Pantev, C., Bertrand, O., Eulitz, C., Verkindt, C., Hampson, S., Schuierer, G., Elbert, T., 1995. Specific tonotopic organizations of different areas of the human auditory cortex revealed by simultaneous magnetic and electric recordings. Electroencephalogr. Clin. Neurophysiol. 94, 26-40.

Pantev, C., Ross, B., Berg, P., Elbert, T., Rockstroh, B., 1998. Study of the human auditory cortices using a whole-head magnetometer: left vs. right hemisphere and ipsilateral vs. contralateral stimulation. Audiol. Neurootol. 3, 183-190.

Reite, M., Adams, M., Simon, J., Teale, P., Sheeder, J., Richardson, D., Grabbe, R., 1994. Auditory M100 component 1: relationship to Heschl's gyri. Brain Res. Cogn. Brain Res. 2, 13-20.

Roberts, T.P., Poeppel, D., 1996. Latency of auditory evoked M100 as a function of tone frequency. Neuroreport 7, 1138-1140.

Rosburg, T., Kreitschmann-Andermahr, I., Emmerich, E., Nowak, H., Sauer, H., 1998. Hemispheric differences in frequency dependent dipole dipole orientation of the human auditory evoked field component N100m. Neurosc. Lett. 258, 105-108.

Schönwiesner, M., Von Cramon, Y., Rübsamen, R., 2002. Is it tonotopy after all. Neuroimage 17, 1144-1161.

Schreiner, C.E., 1998. Spatial distribution of responses to simple and complex sounds in the primary auditory cortex. Audiol. Neurootol. 3, 104-122.

Strainer, J.C., Ulmer, J.L., Yetkin, F.Z., Haughton, V.M., Daniels, D.L., Millen, S.J., 1997. Functional MR of the primary auditory cortex: an analysis of pure tone activation and tone discrimination. AJNR Am. J. Neuroradiol. 18, 601-610.

Szikla, G., Bourvure, G., Hori, T., Petrov, V., 1977. Angiography of the Human Brain Cortex. Springer-Verlag, Berlin.

Verkindt, C., Bertrand, O., Perrin, F., Echallier, J.F., Pernier, J., 1995. Tonotopic organization of the human auditory cortex: N100 topography and multiple dipole model analysis. Electroencephalogr. Clin. Neurophysiol. 96, 143-156.

Wessinger, C.M., Buonocore, M., Kussmaul, C.L., Mangun, G.R., 1997. Tonotopy in human auditory cortex examined with functional magnetic resonance imaging. Human Brain Mapp. 5, 18 25.

Williamson, S.J., Lu, Z.L., Karron, D., Kaufman, L., 1991. Advantages and limitations of magnetic source imaging. Brain Topogr. 4, $169-180$.

Zouridakis, G., Simos, P.G., Papanicolaou, A.C., 1998. Multiple bilaterally asymmetric cortical sources account for the $N 1 \mathrm{~m}$ component. Brain Topogr. 10, 183-189. 